\title{
Instability of nocturnal parasympathetic nerve function in patients with chronic lung disease with or without nocturnal desaturation
}

This article was published in the following Dove Press journal: International Journal of COPD

\section{Keisaku Fujimoto' \\ Haruna Yamazaki² \\ Akikazu Uematsu²}

'Department of Clinical Laboratory Sciences, Shinshu University School of Health Sciences, Matsumoto, Nagano, Japan; ${ }^{2}$ Department of Biomedical Laboratory Sciences, Graduate School of Medicine, Shinshu University, Matsumoto, Nagano, Japan
Correspondence: Keisaku Fujimoto Department of Clinical Laboratory Sciences, Shinshu University School of Health Sciences, 3-I-I Asahi, Matsumoto, Nagano 390-8621, Japan

Tel/fax +8I 263372393

Email keisaku@shinshu-u.ac.jp
Objective/background: This study was performed to evaluate the association of nocturnal autonomic nerve (AN) dysfunction, especially parasympathetic nerve (PN) function instability, and nocturnal oxygen desaturation (NOD) in patients with chronic lung diseases (CLD).

Patients and methods: Twenty-nine stable CLD patients with irreversible pulmonary dysfunction and mild-to-moderate daytime hypoxemia, 13 CLD patients receiving long-term oxygen therapy (LTOT) with maintained $\mathrm{SpO}_{2}>90 \%$, and 17 senior healthy volunteers underwent two-night examinations of nocturnal AN function by pulse rate variability (PRV) instead of heart rate variation using a photoelectrical plethysmograph simultaneously monitoring $\mathrm{SpO}_{2}$ and the presence of sleep disordered breathing at home. AN function was examined by instantaneous time-frequency analysis of PRV using a complex demodulation method.

Results: There were no significant differences in mean low frequency/high frequency (HF) ratio (index of sympathetic nerve activity) or mean $\mathrm{HF}$ amplitude (index of PN activity) among controls and CLD patients with and without NOD (defined as $\mathrm{SpO}_{2}<90 \%$ for at least 3\% of total recording time at night). However, the relative times over which the same main HF peak was sustained for at least 20 seconds $\left(\% \mathrm{HF}_{20 \mathrm{sec}}\right)$ and 5 minutes in total recording time, indexes of PN function stability, were significantly reduced in CLD patients compared with controls, and further decreased in CLD patients with NOD compared with non-NOD. $\% \mathrm{HF}_{20 \mathrm{sec}}$ was significantly higher in the LTOT group than the NOD group. Furthermore, $\mathrm{PaO}_{2}$ at rest and nocturnal hypoxia were significantly correlated with PN function instability in CLD patients.

Conclusion: PN function is unstable at night associated with nocturnal hypoxemia in CLD patients, which may reflect poor quality of sleep.

Keywords: autonomic nerve function, heart rate variability, photoelectric plethysmography, long-term oxygen therapy, sleep disordered breathing

\section{Plain language summary}

Chronic lung disease (CLD) patients show nocturnal parasympathetic nerve (PN) function instability, despite normal sympathetic and PN activity. Decreased PN function stability was associated with nocturnal oxygen desaturation, and was reduced by oxygen therapy. Nocturnal PN function instability associated with nocturnal hypoxemia in CLD patients may correspond to poor quality of sleep.

\section{Introduction}

The prevalence of insomnia is increased in patients with various medical disorders, specifically in asthma and COPD among respiratory diseases, ${ }^{1}$ and it was demonstrated that patients with COPD complained of insomnia and difficulty in sleeping 
and had reduced sleep efficiency, shorter total sleep time, and decreased mean overnight oxygen saturation compared to controls matched for age, gender, and body weight. ${ }^{2}$ It has also been demonstrated that disturbed sleep is crosssectionally associated with worse COPD and is longitudinally predictive of COPD exacerbation, emergency health care utilization, and mortality over 2.4 years of follow-up. ${ }^{3}$ Significant sleep disruption and consequent impairment of physical and social functioning were also demonstrated in patients with idiopathic pulmonary fibrosis (IPF). ${ }^{4}$ Respiratory symptoms, including cough, sputum production, and dyspnea, nicotine use, nicotine withdrawal, increased work of breathing, hypoxemia, hypercapnia, increased sympathetic nerve (SN) activity, comorbid anxiety and depression, comorbid sleep disordered breathing (SDB), restless leg syndrome, and use of medications, such as theophylline, have been suggested as possible etiologies of insomnia in patients with COPD. ${ }^{5,6}$ Among them, nocturnal hypoxia due to hypoventilation and obstructive sleep apnea (OSA) may contribute markedly to nocturnal sleep disturbance. ${ }^{7-9}$ Indeed, nocturnal hypoxemia may be associated with arousals, and lead to sleep fragmentation. ${ }^{7}$ On the other hand, chronic hypoxemia may contribute to autonomic nerve (AN) function, especially SN activation. ${ }^{10-12}$ Increased SN activity may be related to the development of insomnia, which would further contribute to activation of the SN. Thus, it is possible that the synergistic effects of $\mathrm{SN}$ activation due to nocturnal hypoxemia and insomnia in chronic lung disease (CLD), including COPD, may contribute to some of the adverse outcomes. Oxygen use was found to be associated with lower rates of insomnia. ${ }^{13}$ However, conflicting data have been reported regarding the role of oxygen in improving quality of sleep in COPD; while some studies demonstrated a beneficial effect of supplemental oxygen, ${ }^{13,14}$ others did not. ${ }^{15,16}$

Analysis of heart rate variability (HRV) is a simple and noninvasive method to assess AN function, generally evaluated by power spectrum analysis of HRV. ${ }^{17,18}$ The ratio of the power of low-frequency (LF) bundle and high-frequency (HF) bundle, that is, the LF/HF ratio, represents SN activity and the power of HF represents parasympathetic nerve (PN) activity. Recently, it was demonstrated that instantaneous time-frequency analysis of HRV using a complex demodulation (CD) method ${ }^{19,20}$ allows estimation of transitional changes of $\mathrm{PN}$ function. ${ }^{21} \mathrm{We}$ have reported the modulation effects of disordered breathing on HF bundle and showed that the stability of PN function during sleep was impaired in patients with OSA. ${ }^{22}$ This study was performed to clarify the presence of nocturnal AN dysfunction in association with nocturnal hypoxemia in CLD, including COPD.

\section{Patients and methods}

\section{Subjects}

Twenty-nine patients with stable CLD who showed irreversible pulmonary dysfunction characterized by FVC $<80 \%$ and/or $\mathrm{FEV}_{1} / \mathrm{FVC}<70 \%$ and/or carbon monoxide diffusion capacity $<80 \%$ and mild-to-moderate daytime hypoxemia $>60$ Torr at rest, consisting of 22 patients with COPD, two patients with combined pulmonary fibrosis and emphysema (CPFE), two patients with IPF, and three patients with bronchiectasis (BE), were recruited from the outpatient clinic of Shinshu University Hospital between September 2012 and May 2017 (Table 1). Furthermore, 13 patients with CLD who were treated with long-term oxygen therapy (LTOT) and maintained $\mathrm{PaO}_{2}>60$ Torr at night, excluding patients who showed nocturnal oxygen desaturation (NOD), consisting of eight with COPD, two with CPFE, two with IPF, and one with BE, were also recruited. NOD was defined as percutaneous oxygen saturation $\left(\mathrm{SpO}_{2}\right)<90 \%$ lasting for at least $3 \%$ of the total recording time during the night. Seventeen senior healthy volunteers $>60$ years old without

Table I Characteristics and results of pulmonary function test and arterial blood gas analysis in healthy elderly subjects (control group) and chronic lung disease patients with ventilatory disturbance with and without NOD (NOD group and non-NOD group, respectively), and who have been treated with LTOT (LTOT group)

\begin{tabular}{|c|c|c|c|c|}
\hline & Control & non-NOD & NOD & LTOT \\
\hline Number & 17 & 17 & 12 & 13 \\
\hline COPD, n & & 12 & 10 & 8 \\
\hline CPFE, $n$ & & 1 & I & 2 \\
\hline IP, n & & 2 & 0 & 2 \\
\hline $\mathrm{BE}, \mathrm{n}$ & & 2 & I & I \\
\hline Age, years & $65.3 \pm 1.7$ & $77.0 \pm 2.3 * *$ & $76.4 \pm 3.3 * *$ & $77.2 \pm 2.2 * *$ \\
\hline Sex, male/female & $15 / 2$ & $15 / 2$ & $12 / 0$ & $13 / 0$ \\
\hline $\mathrm{BMI}, \mathrm{kg} / \mathrm{m}^{2}$ & $23.4 \pm 0.7$ & $22.5 \pm 0.9$ & $20.6 \pm 1.0 *$ & $21.7 \pm 0.9$ \\
\hline FVC, \% & $103.2 \pm 1.8$ & $98.7 \pm 6.0$ & $93.1 \pm 6.2$ & $73.4 \pm 5.1^{* * \ldots \#, \S}$ \\
\hline $\mathrm{FEV}_{1}, \%$ & $97.6 \pm 2.3$ & $74.2 \pm 4.2 * *$ & $54.8 \pm 6.1 * *, \#$ & $45.4 \pm 5.5^{* *}$ \\
\hline $\mathrm{FEV}_{1} / \mathrm{FVC}, \%$ & $78.6 \pm 1.7$ & $61.9 \pm 3.6 * *$ & $46.2 \pm 4.3^{* * * \#}$ & $51.0 \pm 6.8 * *$ \\
\hline FRC, \% & & $102.0 \pm 5.7$ & $103.8 \pm 7.1$ & $122.4 \pm 12.3$ \\
\hline $\mathrm{RV}, \%$ & & $142.5 \pm 13.2$ & $171.7 \pm 19.7$ & $203.0 \pm 30.9$ \\
\hline TLC, \% & & II $3.8 \pm 6.0$ & I $18.3 \pm 7.0$ & $121.1 \pm 10.9$ \\
\hline RV/TLC, \% & & $43.8 \pm 2.6$ & $48.4 \pm 3.7$ & $54.8 \pm 4.3^{\#}$ \\
\hline DLCO, \% & & $67.7 \pm 4.9$ & $46.6 \pm 4.4^{\# \#}$ & $34.1 \pm 3.1^{\# \#}$ \\
\hline $\mathrm{PaO}_{2}$, Torr & & $75.9 \pm 2.5$ & $66.0 \pm 2.4^{\#}$ & $72.6 \pm 3.6$ \\
\hline $\mathrm{PaCO}_{2}$, Torr & & $38.4 \pm 1.0$ & $36.6 \pm 1.8$ & $42.4 \pm 1.3^{\#, 8}$ \\
\hline $\mathrm{HCO}_{3}^{-}, \mathrm{mmol} / \mathrm{L}$ & & $24.6 \pm 0.4$ & $24.0 \pm 0.9$ & $26.5 \pm 0.7^{\#}$ \\
\hline
\end{tabular}

Notes: Values are means \pm SEM. $* P<0.05$ and $* * P<0.01$ vs control subjects; ${ }^{*} P<0.05$ and ${ }^{\#} P<0.0$ I vs non-NOD; $\$$ P $<0.05$ vs NOD. NOD was defined as $C T 90 \geq 3 \%$. Abbreviations: CPFE, combined pulmonary fibrosis and emphysema; IP, interstitial pneumonia; $\mathrm{BE}$, bronchiectasis; $\mathrm{BMI}$, body mass index; FRC, functional residual capacity; RV, residual volume; TLC, total lung capacity; DLCO, carbon monoxide diffusing capacity; LTOT, long-term oxygen therapy; NOD, nocturnal oxygen desaturation; SEM, standard error of means; CT90, cumulative time with $\mathrm{SpO}_{2}<90 \%$. 
any daytime sleepiness or sleep disorders, and with normal spirometry and respiratory event index $(\mathrm{REI})<10$ events/h were also recruited as control subjects. Subjects who showed arrhythmia $>10 \%$ of the total pulse rate and atrial fibrillation and were diagnosed with diabetes mellitus, heart failure, renal failure, or impaired cognitive function by a medical specialist were excluded from the study. This study was conducted in accordance with the International Conference on Harmonization-Good Clinical Practice and the Declaration of Helsinki (2008), and was approved by the institutional research ethics committee of Shinshu University School of Medicine (No 2100). All subjects were given an adequate explanation of the study and provided written informed consent.

\section{Methods}

All subjects underwent a two-night study at home. SDB was monitored using a portable monitoring device (Sleep Eye; Denso Corporation, Kariya, Japan). $\mathrm{SpO}_{2}$ was recorded continuously by pulse oximetry (Pulsox DS-Me; Teijin Co. Ltd., Tokyo, Japan), and the peak-to-peak intervals (PPI) of volume pulse wave were recorded overnight using a photoelectrical plethysmograph (Denso Corporation) attached to the wrist. Nocturnal AN functions were evaluated by instantaneous time-frequency analysis of pulse rate variability (PRV) using a CD method. ${ }^{19,20}$ Patients with CLD without LTOT were divided into two subgroups according to the presence or absence of NOD, and comparisons were performed among the control subjects, CLD with and without NOD, and CLD treated with LTOT.

\section{Evaluation of SDB}

The presence of SDB was determined using a portable monitoring device (Sleep Eye; Denso Corporation), which is a nonrestrictive and seat-type device $(812 \mathrm{~mm}$ [W] $\times 555 \mathrm{~mm}$ [D] $\times 33 \mathrm{~mm}[\mathrm{H}]$ ), and is used for screening of SDB in Japan. ${ }^{23}$ Ninety film-type pressure-sensitive sensors were used to convert body pressure changes with breathing into a breathing wave pattern. The portable monitoring device was spread under the bedsheet, and the data were recorded on flash memory and analyzed by specialized software.

\section{Analysis of AN function}

PRV in the frequency domain detected using a photoelectric plethysmograph instead of HRV analyzed from electrocardiography was evaluated for analysis of AN function as described in our previous report. ${ }^{22}$ The PRV technique using the same device was already validated against traditional HRV. ${ }^{24}$ The sampling frequency of the electric plethysmograph was $20 \mathrm{~Hz}$. When the PPI of the volume pulse wave due to isolated/sporadic premature supraventricular/ventricular contraction or artifact was markedly different from those just before and after PPI, it was eliminated from the analysis automatically. PRV was evaluated by instantaneous time-frequency analysis using the CD method. ${ }^{19,20}$ The frequency spectra of the PPI data were estimated for the range between 0 and $0.40 \mathrm{~Hz}$ and divided into two components depending on their central frequencies (CF); one domain between 0.04 and $0.15 \mathrm{~Hz}$ was labeled as the band with LF and the other between 0.15 and $0.40 \mathrm{~Hz}$ as the band with HF. The LF/HF ratio was used as a marker of $\mathrm{SN}$ discharge to the cardiac sinus node. ${ }^{17}$ The mean values of $\mathrm{HF}$ amplitude were used as markers of PN discharge. ${ }^{24}$ Furthermore, nocturnal PN dysfunction was also evaluated as the stability of PN discharge according to the method reported by Yamaguchi et $\mathrm{al}^{21}$ using HRV LOG Analysis Pro-DSA software (NoruPro Light Systems Inc., Tokyo, Japan). Briefly, the HF domain with the maximum instantaneous amplitude was defined as the main HF peak and was used as a surrogate marker of PN discharge. The shift in CF of the main HF peak over time was monitored continuously based on the density spectrum array (DSA) map for the main HF peak constructed with a time scale of 1 second and a frequency resolution of $0.002 \mathrm{~Hz}$. When the CF of the main HF peak was shifted by more than $\pm 0.014 \mathrm{~Hz}$, corresponding to approximately $\pm 5 \%$ of that of respiratory sinus arrhythmia, we assumed that the PN discharge was significantly altered from the previous state, that is, disruption of the PN discharge. When the main HF peak lasted for at least 20 seconds or 5 minutes without any disruption on the HF-DSA map, the PN function was considered to be stable or very stable. As an index of PN function stability, we calculated the relative times over which the same main HF peak was sustained for at least 20 seconds and 5 minutes in total recording, represented as $\% \mathrm{HF}_{20 \mathrm{sec}}$ and $\% \mathrm{HF}_{5 \min }$, respectively.

\section{Data analysis}

Data were obtained on two nights in each subject, and displayed with the mean of two nights. Values in the text, tables, and figures are shown as means \pm standard error of the mean (SEM). The Mann-Whitney $U$ test was used for comparison of variables among groups. Spearman's rank correlation coefficient was used for bivariate correlation analysis. Multiple stepwise linear regression analysis was performed to identify which variables were significant determinants for AN activity and $\mathrm{PN}$ instability. A value of $P \leq 0.15$ was used to identify candidate variables, and then variables were removed from the regression model if $P>0.1$. All statistical analyses were performed using StatFlex version 6 (Artech Co., Ltd., Osaka, Japan). In all analyses, $P<0.05$ was considered to indicate statistical significance. 


\section{Results}

Table 1 shows the constitution of CLD, age, sex, body mass index (BMI), pulmonary function, and arterial blood gas analysis among the CLD patients with and without NOD (NOD group and non-NOD group, respectively), and CLD patients treated with long-term oxygen (LTOT group). Patients in the NOD group showed significantly more severe airflow obstruction and lower carbon monoxide diffusing capacity (DLCO) and $\mathrm{PaO}_{2}$ than those in the non-NOD group. Patients in the LTOT group showed decreased FVC, the most severe airflow obstruction, and the lowest DLCO. The results of arterial blood gas analysis in the LTOT group were obtained under conditions of oxygen inhalation and showed significant increases in mean $\mathrm{PaCO}_{2}$ and bicarbonate levels. Table 2 shows the sleep study results. REI in the non-NOD group was significantly increased compared with control subjects, but there were no significant differences among the three CLD groups. In addition, there were no significant differences in the prevalence of SDB showing REI $\geq 15$ events/h. Patients in the NOD group showed marked nocturnal hypoxemia and increased cumulative time with $\mathrm{SpO}_{2}<90 \%$ (CT90). On the other hand, in the LTOT group, all patients showed CT90 <3\% and maintained oxygenation.

Table 2 Results of nocturnal respiratory events, oxygen desaturation, and autonomic nerve function in healthy elderly subjects (control) and chronic lung disease patients with ventilatory disturbance with and without NOD (NOD group and non-NOD group, respectively), and who have been treated with LTOT (LTOT group)

\begin{tabular}{|c|c|c|c|c|}
\hline & Control & non-NOD & NOD & LTOT \\
\hline Number & 17 & 17 & 12 & 13 \\
\hline REl, events/h & $8.9 \pm 0.9$ & $18.4 \pm 3.0^{*}$ & $12.9 \pm 2.1$ & $11.5 \pm 1.9$ \\
\hline $\begin{array}{l}\mathrm{REI} \geq 15 \text { events } / \mathrm{h}, \\
\mathrm{n}(\%)\end{array}$ & $0(0)$ & $9(52.9)$ & $6(50.0)$ & $5(38.5)$ \\
\hline Mean $\mathrm{SpO}_{2}, \%$ & $94.9 \pm 0.4$ & $95.5 \pm 0.4$ & $91.2 \pm 0.5^{* * \ldots \#}$ & $96.7 \pm 0.4^{* *, 5 \S}$ \\
\hline Lowest $\mathrm{SpO}_{2}, \%$ & $86.1 \pm 1.3$ & $87.9 \pm 1.1$ & $76.8 \pm 2.5^{\text {**. } \ldots}$ & $89.5 \pm 1.1^{* \$ \$}$ \\
\hline СТ $90, \%$ & $2.5 \pm 1.0$ & $0.8 \pm 0.2$ & $27.7 \pm 6.0$ **\# & $0.4 \pm 0.2^{*, 85}$ \\
\hline $3 \%$ ODI, events/h & $7.7 \pm 1.5$ & $4.4 \pm 2.1^{*}$ & $11.0 \pm 2.5^{\# \#}$ & I.I $\pm 0.3^{* *, 8 \S}$ \\
\hline $4 \%$ ODI, events/h & $4.7 \pm 1.0$ & $3.2 \pm 1.6$ & $6.5 \pm 1.6^{\#}$ & $0.5 \pm 0.2^{* *, \$ \S}$ \\
\hline LF/HF ratio & $0.97 \pm 0.06$ & $0.96 \pm 0.09$ & $0.88 \pm 0.09$ & $0.74 \pm 0.04^{* *}$ \\
\hline HFamp, ms & $21.8 \pm 1.9$ & $27.1 \pm 4.2$ & $20.7 \pm 1.8$ & $24.0 \pm 2.1$ \\
\hline $\mathrm{HF}_{20 \mathrm{sec}^{\prime}} \%$ & $51.7 \pm 4.0$ & $25.9 \pm 2.9 * *$ & $15.2 \pm 1.9$ **.\# & $32.2 \pm 5.0 * * .55$ \\
\hline $\mathrm{HF}_{5 \text { min }}, \%$ & $19.5 \pm 3.8$ & $2.5 \pm 1.2^{* *}$ & $0.2 \pm 0.1 * *$ & $2.9 \pm 1.7 * *$ \\
\hline
\end{tabular}

Notes: Values are means \pm SEM. $* P<0.05$ and $* * P<0.01$ vs control subjects; ${ }^{\#} P<0.05$ and ${ }^{\# P} P 0.01$ vs non-NOD; ${ }^{\$} P<0.05$ and ${ }^{\$} \$ P<0.01$ vs NOD. NOD was defined as CT90 $\geq 3 \%$.

Abbreviations: REl, respiratory event index; CT90, cumulative time with $\mathrm{SpO}_{2}<90 \%$; ODI, oxygen desaturation index; LF, low frequency; HF, high frequency; $\mathrm{LF} / \mathrm{HF}$ ratio, a marker of sympathetic nerve activity; HFamp, mean HF amplitude (a marker of parasympathetic nerve activity); $\mathrm{HF}_{20 \sec }$ and $\mathrm{HF}_{5 \min }$, the relative times over which the same main HF peak was sustained for at least 20 seconds and 5 minutes in total recording time (markers of stability of parasympathetic nerve function); LTOT, long-term oxygen therapy; NOD, nocturnal oxygen desaturation; SEM, standard error of means.
With regard to AN function, there were no significant differences in mean LF/HF ratio, an index of SN activity, or the mean HF amplitude, an index of PN activity, among control subjects and CLD patients with and without NOD (Figure 1). However, the mean LF/HF ratio in the LTOT group was significantly lower than that in the controls. On the other hand, $\% \mathrm{HF}_{20 \mathrm{sec}}$ and $\% \mathrm{HF}_{5 \text { min }}$, indexes of the stability of PN function, showed significant decreases in CLD patients in non-NOD and NOD groups compared with controls, and $\% \mathrm{HF}_{20 \text { sec }}$ in the NOD group was further reduced compared with the non-NOD group (Figure 2). In the LTOT group, both $\% \mathrm{HF}_{20 \sec }$ and $\% \mathrm{HF}_{5 \min }$ were significantly decreased compared to those in the controls; however, $\% \mathrm{HF}_{20 \text { sec }}$ showed significantly higher values compared with the NOD group. There were significant correlations between $\% \mathrm{HF}_{20 \mathrm{sec}}$ and CT90 $(r=-0.41, P<0.05)$ or $\mathrm{PaO}_{2}$ at rest $(r=0.58, P<0.01)$ in CLD patients in non-NOD and NOD groups (Figure 3). In CLD patients in the non-NOD and NOD groups, multiple regression analyses among age, sex, BMI, pulmonary function test, blood gas analysis, CT90, 3\% oxygen desaturation index, and REI revealed that the regression model for $\% \mathrm{HF}_{20 \mathrm{sec}}$ was significant $(r=0.76, P<0.0001)$ and consisted of BMI $(\operatorname{std} \beta=0.29, P=0.0499), \mathrm{CT} 90(\operatorname{std} \beta=-0.39, P=0.0109), \mathrm{PaO}_{2}$ $(\operatorname{std} \beta=0.65, P=0.0006), \% \mathrm{FEV}_{1}(\operatorname{std} \beta=-0.40, P=0.05307)$. $\mathrm{PaO}_{2}$ at rest was the most significant determinant.

\section{Discussion}

Nocturnal AN function was evaluated by instantaneous time-frequency analysis of PRV using a CD method in 29 CLD patients with and without NOD and 13 CLD patients treated with LTOT. There were no significant differences in $\mathrm{SN}$ or PN activity among the groups. However, the indexes of stability of PN function showed significant decreases in CLD patients compared with control subjects, and were further decreased in CLD patients with NOD compared to the nonNOD group. The instability of PN function was significantly associated with oxygenation at rest and nocturnal hypoxemia. These findings suggest that PN function is unstable in CLD patients associated with nocturnal hypoxemia.

It has been suggested that $\mathrm{SN}$ activity is increased in patients with COPD because muscle sympathetic activity assessed by microneurography is increased and decreases with short-term oxygen supplementation, ${ }^{25}$ and plasma norepinephrine levels are elevated in hypoxemic patients with COPD, and the levels decrease with LTOT. ${ }^{26}$ Hypoxemia, especially chronic hypoxemia, and hypercapnia have been shown to increase SN activity. ${ }^{11,27,28}$ However, in this study, CLD patients did not show any increases in SN activity even 
A

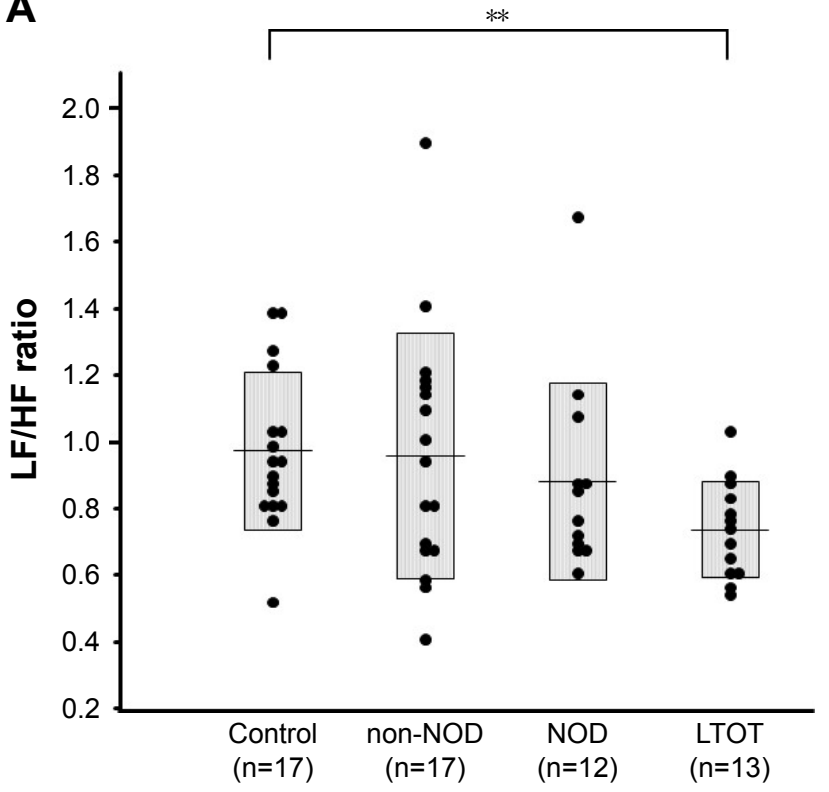

B

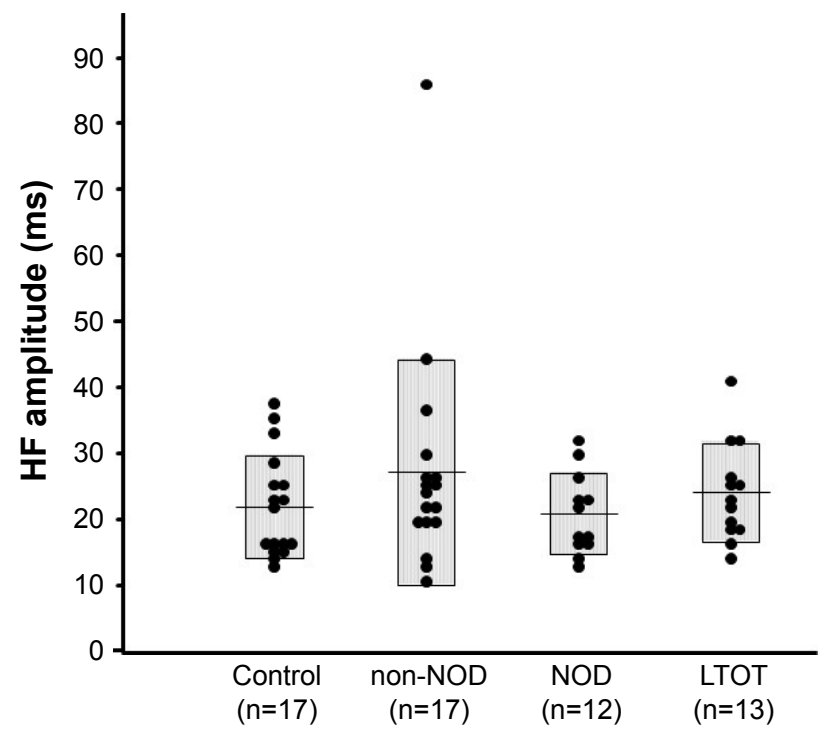

Figure I Comparison of sympathetic (A) and parasympathetic (B) activity among healthy elderly subjects (control), CLD patients with NOD and without NOD (non-NOD), and CLD patients treated with LTOT.

Note: $* * P<0.01$ vs control.

Abbreviations: LF, low frequency; HF, high frequency; LF/HF ratio, an index of sympathetic nerve activity; HF amplitude, an index of parasympathetic nerve activity; NOD, nocturnal oxygen desaturation; LTOT, long-term oxygen therapy; CLD, chronic lung disease.

though they were divided into two groups with and without NOD, although the SN activity was suppressed by LTOT. The results were not expected. We hypothesized that the $\mathrm{LF} / \mathrm{HF}$ ratio will be increased and the mean HF amplitude will be suppressed, and the stability of PN function will be impaired especially in the CLD patients with NOD. However, there were no differences in the marker of SN activity, LF/ $\mathrm{HF}$ ratio, and marker of PN activity, mean HF amplitude,
A

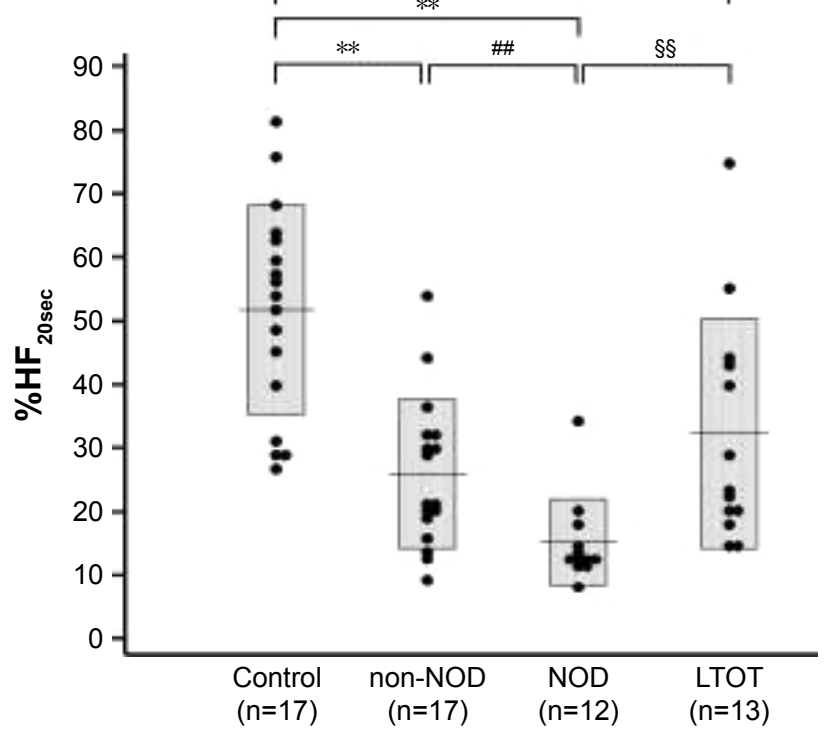

B

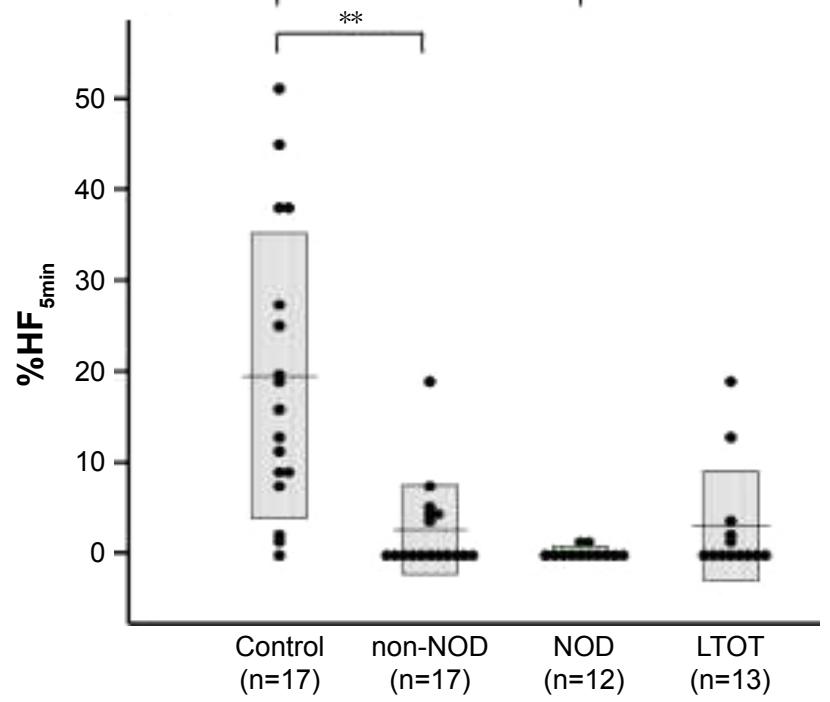

Figure 2 Comparison of indexes of stability of parasympathetic nerve function, $\% \mathrm{HF}_{20 \sec }(\mathbf{A})$ and $\% \mathrm{HF}_{5 \min }$ (B), among healthy elderly subjects (control), CLD patients with NOD and without NOD (non-NOD), and CLD patients treated with LTOT.

Notes: ${ }^{* * P}<0.01$ vs control; ${ }^{\#} P<0.01$ vs non-NOD; ${ }^{\S} P<0.01$ vs NOD.

Abbreviations: $\% \mathrm{HF}_{20 \text { sec }}$ and \% $\mathrm{HF}_{5 \min }$, the relative times over which the same main high-frequency peak was sustained for at least 20 seconds and 5 minutes in total recording time (markers of stability of parasympathetic nerve function); CLD, chronic lung disease with irreversible pulmonary dysfunction and mild-to-moderate daytime hypoxemia; NOD, nocturnal oxygen desaturation defined as CT90 $\geq 3 \%$; CT90, cumulative time with $\mathrm{SpO}_{2}<90 \%$; LTOT, long-term oxygen therapy. 

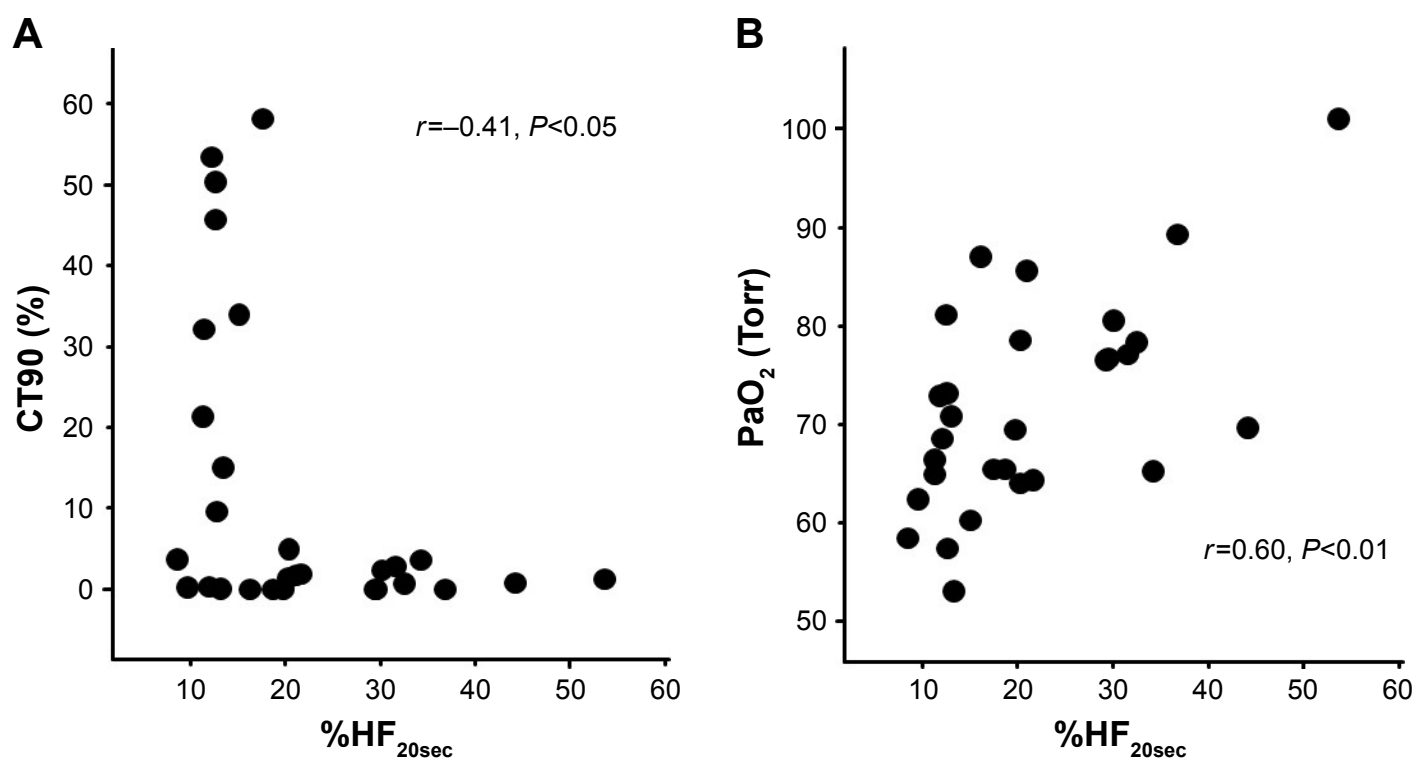

Figure 3 Relationship between $\% \mathrm{HF}_{20 \text { sec }}$, an index of stability of parasympathetic nerve function, and $\mathrm{CT} 90(\mathbf{A})$ or $\mathrm{PaO}$ (B).

Abbreviations: $\mathrm{CT} 90$, cumulative time with $\mathrm{SpO}_{2}<90 \%$; $\% \mathrm{HF}_{20 \text { sec }}$, the relative time over which the same main $\mathrm{HF}$ peak was sustained for at least 20 seconds in total recording time (an index of stability of parasympathetic nerve function); HF, high frequency.

from those in elderly healthy subjects whereas the stability of PN function was impaired. Analysis of HRV is a simple and noninvasive method to assess AN function. However, consistent results could not be obtained in previous research regarding SN activity evaluated by HRV. In the present study, we could not obtain expected results, although we used an instantaneous time-frequency analysis by the method of CD, which was different from the methods previously reported and improved time resolution. One reason may be that the cardiovascular baroreceptor reflex is suppressed in elderly people. In addition, COPD patients show depressed HRV in association with systemic inflammation and lung function impairment. ${ }^{29,30}$ Another reason may be that the frequency analysis of HRV, especially very LF and LF bundle, are affected by the cyclic variation of heart rate due to repeated periods of apnea and hypopnea. ${ }^{14,15}$

Recently, it has been demonstrated that the instantaneous time-frequency analysis of HRV using the $\mathrm{CD}$ method allows estimation of transitional changes of $\mathrm{PN}$ function during sleep in patients with OSA. ${ }^{21}$ Yamaguchi et $\mathrm{al}^{21}$ and $\mathrm{we}^{22}$ used this method and reported that the stability of PN function during sleep in OSA was impaired but improved following treatment with continuous positive airway pressure. We also demonstrated that the instability of PN function was closely associated with arousal caused by apnea and hypopnea, ${ }^{22}$ and may be useful to evaluate sleep quality. The instability of PN function was also demonstrated in patients with CLD, associated with daytime $\mathrm{PaO}_{2}$ at rest and NOD, and was reduced by LTOT. This is the first study to evaluate the stability of PN function in patients with CLD. Some COPD patients show deterioration of sleep efficiency, and it has been suggested that nocturnal hypoxemia may be associated with arousals and leads to sleep fragmentation. ${ }^{8}$ Therefore, it is speculated that sleep fragmentation and frequent arousals may cause instability of PN function, represented by modulation of the HF bundle power spectrum. In this study, a significant and reasonable association with pulmonary dysfunction and AN function was not obtained. Some studies suggested worse sleep in more severe COPD,${ }^{15}$ while other studies have not shown an association between $\mathrm{FEV}_{1}$ and reported sleep quality., ${ }^{3,5}$ On the other hand, CLD is a state of chronic systemic inflammation, and the pro-inflammatory state has been demonstrated to be associated with AN dysfunction ${ }^{31}$ and to modulate airway inflammation. ${ }^{32}$ There is a possibility that the AN dysfunction induced by chronic airway or systemic inflammation may be associated with the instability of PN function.

Supplemental oxygen may help to improve sleep quality in COPD patients with nocturnal hypoxemia. ${ }^{13}$ However, data on the role of oxygen in improving sleep in COPD have been conflicting, because supplemental oxygen aggravated hypoventilation and resulted in hypercapnia. ${ }^{13-16}$ In this study, the CLD patients who were prescribed LTOT and maintained oxygenation during night showed lower SN activity and significant reduced PN instability. Thirty-one percent of the patients showed mild hypercapnia $\left(\mathrm{PaCO}_{2}<50\right.$ Torr). Therefore, nocturnal hypoxemia may be an important contributing factor for PN instability. OSA may be present at a rate of $\sim 10 \%-30 \%$ in persons with COPD,${ }^{31}$ which may 
affect AN function. In this study, half of the patients showed $\mathrm{REI} \geq 15$ events/ $h$, but there were no significant differences in prevalence among the groups. SDB may contribute to PN instability; however, a significant association with SDB and the instability of PN function could not be obtained.

This study had some limitations. First, sleep quality and sleep apnea and hypopnea were not evaluated by polysomnography (PSG). When the patients prescribed LTOT are examined by PSG, oxygen supply should be stopped, which will induce severe hypoxemia. Therefore, it is ethically difficult to perform PSG examination in patients with CLD prescribed LTOT. However, simpler electroencephalography should be performed to clarify the association with arousals and the instability of PN function.

In conclusion, against our expectations, we could not show any increase in SN activity in CLD patients. However, $\mathrm{PN}$ function evaluated by the instantaneous time-frequency analysis of PRV using a CD method is unstable in CLD patients associated with nocturnal hypoxemia. The instability of PN function may be reduced by supplemental oxygen. The instability may reflect insomnia or poor quality of sleep, which may be related with frequent arousal and sleep fragmentation due to NOD in patients with CLD.

\section{Acknowledgments}

We thank Toshiro Momose and technicians of polysomnography for their help and support. This study was supported by a Grant-in-Aid for Scientific Research Expenses from The Ministry of Education, Culture, Sports, Science and Technology, Japan (15K01281).

\section{Disclosure}

The authors report no conflicts of interest in this work.

\section{References}

1. Valipour A, Lavie P, Lothaller H, Mikulic I, Burghuber OC. Sleep profile and symptoms of sleep disorders in patients with stable mild to moderate chronic obstructive pulmonary disease. Sleep Med. 2011; 12(4):367-372.

2. Budhiraja R, Roth T, Hudgel DW, Budhiraja P, Drake CL. Prevalence and polysomnographic correlates of insomnia comorbid with medical disorders. Sleep. 2011;34(7):859-867.

3. Omachi TA, Blanc PD, Claman DM, et al. Disturbed sleep among COPD patients is longitudinally associated with mortality and adverse COPD outcomes. Sleep Med. 2012;13(5):476-483.

4. Mermigkis C, Stagaki E, Amfilochiou A, et al. Sleep quality and associated daytime consequences in patients with idiopathic pulmonary fibrosis. Med Princ Pract. 2009;18(1):10-15.

5. Budhiraja R, Parthasarathy S, Budhiraja P, Habib MP, Wendel C, Quan SF. Insomnia in patients with COPD. Sleep. 2012;35(3):369-375.

6. Budhiraja R, Siddiqi TA, Quan SF. Sleep disorders in chronic obstructive pulmonary disease: etiology, impact, and management. J Clin Sleep Med. 2015;11(3):259-270.
7. Owens RL. Supplemental oxygen needs during sleep. Who benefits? Respir Care. 2013;58(1):32-47.

8. Cormick W, Olson LG, Hensley MJ, Saunders NA. Nocturnal hypoxaemia and quality of sleep in patients with chronic obstructive lung disease. Thorax. 1986;41(11):846-854.

9. Flenley DC. Sleep in chronic obstructive lung disease. Clin Chest Med. 1985;6(4):651-661.

10. Sica AL, Greenberg HE, Ruggiero DA, Scharf SM. Chronic-intermittent hypoxia: a model of sympathetic activation in the rat. Respir Physiol. 2000;121(2-3):173-184.

11. Calbet JA. Chronic hypoxia increases blood pressure and noradrenaline spillover in healthy humans. $J$ Physiol. 2003;551(Pt 1): 379-386.

12. Hardy JC, Gray K, Whisler S, Leuenberger U. Sympathetic and blood pressure responses to voluntary apnea are augmented by hypoxemia. J Appl Physiol. 1994;77(5):2360-2365.

13. Calverley PM, Brezinova V, Douglas NJ, Catterall JR, Flenley DC. The effect of oxygenation on sleep quality in chronic bronchitis and emphysema. Am Rev Respir Dis. 1982;126(2):206-210.

14. Goldstein RS, Ramcharan V, Bowes G, Mcnicholas WT, Bradley D, Phillipson EA. Effect of supplemental nocturnal oxygen on gas exchange in patients with severe obstructive lung disease. $N$ Engl $J$ Med. 1984;310(7):425-429.

15. Fleetham J, West P, Mezon B, Conway W, Roth T, Kryger M. Sleep, arousals, and oxygen desaturation in chronic obstructive pulmonary disease. The effect of oxygen therapy. Am Rev Respir Dis. 1982; 126(3):429-433.

16. Mckeon JL, Murree-Allen K, Saunders NA. Supplemental oxygen and quality of sleep in patients with chronic obstructive lung disease. Thorax. 1989;44(3):184-188.

17. Malliani A, Pagani M, Lombardi F, Cerutti S. Cardiovascular neural regulation explored in the frequency domain. Circulation. 1991; 84(2):482-492.

18. Tobaldini E, Nobili L, Strada S, Casali KR, Braghiroli A, Montano N. Heart rate variability in normal and pathological sleep. Front Physiol. 2013;4:294.

19. Hayano J, Taylor JA, Yamada A, et al. Continuous assessment of hemodynamic control by complex demodulation of cardiovascular variability. Am J Physiol. 1993;264(4 Pt 2):H1229-H1238.

20. Hayano J, Taylor JA, Mukai S, et al. Assessment of frequency shifts in R-R interval variability and respiration with complex demodulation. J Appl Physiol. 1994;77(6):2879-2888.

21. Yamaguchi K, Ohki N, Kobayashi M, et al. Estimation of parasympathetic nerve function during sleep in patients with obstructive sleep apnea by instantaneous time-frequency analysis. Sleep Med. 2014; 15(1):33-41.

22. Fujimoto K, Ura M, Yamazaki H, Uematsu A. Instability of parasympathetic nerve function evaluated by instantaneous time-frequency analysis in patients with obstructive sleep apnea. Sleep Biol Rhythms. 2018;16(3):323-330.

23. Agatsuma T, Fujimoto K, Komatsu Y, et al. A novel device (SD-101) with high accuracy for screening sleep apnoea-hypopnoea syndrome. Respirology. 2009;14(8):1143-1150.

24. Hayano J, Barros AK, Kamiya A, Ohte N, Yasuma F. Assessment of pulse rate variability by the method of pulse frequency demodulation. Biomed Eng Online. 2005;4:62.

25. Heindl S, Lehnert M, Criée CP, Hasenfuss G, Andreas S. Marked sympathetic activation in patients with chronic respiratory failure Am J Respir Crit Care Med. 2001;164(4):597-601.

26. Bratel T, Wennlund A, Carlström K. Impact of hypoxaemia on neuroendocrine function and catecholamine secretion in chronic obstructive pulmonary disease (COPD). Effects of long-term oxygen treatment. Respir Med. 2000;94(12):1221-1228.

27. Oikawa S, Hirakawa H, Kusakabe T, Nakashima Y, Hayashida Y. Autonomic cardiovascular responses to hypercapnia in conscious rats: the roles of the chemo- and baroreceptors. Auton Neurosci. 2005;117(2): 105-114. 
28. Gilmartin GS, Lynch M, Tamisier R, Weiss JW. Chronic intermittent hypoxia in humans during 28 nights results in blood pressure elevation and increased muscle sympathetic nerve activity. Am J Physiol Heart Circ Physiol. 2010;299(3):H925-H931.

29. Corbo GM, Inchingolo R, Sgueglia GA, Lanza G, Valente S. C-reactive protein, lung hyperinflation and heart rate variability in chronic obstructive pulmonary disease - a pilot study. COPD. 2013;10(2): 200-207.
30. van Gestel AJ, Kohler M, Steier J, et al. Cardiac autonomic function and cardiovascular response to exercise in patients with chronic obstructive pulmonary disease. COPD. 2012;9(2):160-165.

31. van Gestel AJ, Steier J. Autonomic dysfunction in patients with chronic obstructive pulmonary disease (COPD). J Thorac Dis. 2010;2(4):215-222.

32. Mcgovern AE, Mazzone SB. Neural regulation of inflammation in the airways and lungs. Auton Neurosci. 2014;182:95-101.

\section{Publish your work in this journal}

The International Journal of COPD is an international, peer-reviewed journal of therapeutics and pharmacology focusing on concise rapid reporting of clinical studies and reviews in COPD. Special focus is given to the pathophysiological processes underlying the disease, intervention programs, patient focused education, and self management protocols.

\section{Dovepress}

This journal is indexed on PubMed Central, MedLine and CAS. The manuscript management system is completely online and includes a very quick and fair peer-review system, which is all easy to use. Visit http://www.dovepress.com/testimonials.php to read real quotes from published authors.

Submit your manuscript here: http://www.dovepress.com/international-journal-of-chronic-obstructive-pulmonary-disease-journal 